\title{
Ökologischer Anpassungsdruck
}

\section{Der Osterweiterungsprozeß der Europäischen Union geht in eine wichtige Phase: Die von der Kommission entworfene Vorbereitungsstrategie Agenda 2000 soll im März beschlossen werden. Über die Finanzierungsdebatte werden jedoch die inhaltlichen Kernpunkte vernachlässigt. Die bereits laufenden Bei- trittsverhandlungen haben auch eine wichtige ökologische Dimension.}

$\mathrm{A}$

Von Jan Nill und Ulrich Petschow Is eine wesentliche Voraussetzung für den Beitritt der mittel- und osteuropäischen Länder (MOEL) Polen, Tschechien, Ungarn, Slowenien und Estland wird von Seiten der EU die Übernahme des ,acquis communautaire“ (AC), also des gemeinschaftlichen Besitzstandes angesehen. Dies gilt auch für den Umweltbereich. Im Vergleich zur Süderweiterung Anfang der 80er Jahre, die ebenfalls Beitrittsländer mit deutlich niedrigerem Einkommensniveau betraf - sind allerdings zumindest zwei wesentliche Unterschiede festzuhalten:

Zum einen hat sich der AC im Umweltbereich deutlich weiterentwickelt; damit haben sich jedoch auch die Anpassungskosten erhöht.

- Zum anderen haben sich die Verteilungspositionen deutlich verändert. Die derzeitigen Mitgliedstaaten sind nicht mehr zu einer finanziellen Unterstïtzung der Annäherung an den AC in ähnlichem Umfang wie damals bereit.

Die Übernahme des AC im Umweltbereich wird dennoch gefordert, um eine Störung des Binnenmarktes zu verhindern und protektionistische Tendenzen zu vermeiden (1). Das umweltrechtliche Regelwerk der der EU umfaßt heute etwa 300 Rechtsakte. Etwa die Hälfte davon entfällt auf produktbezogene Regelungen. Diese stellen den unmittelbar binnenmarktrelevanten Teil des AC dar und müssen von den MOEL bereits zum Beitrittszeitpunkt übernommen werden. Für die Umsetzung wichtiger anderer umweltpolitischer Regulierungen z.B. zur Luftqualität, zur Umweltverträglichkeitsprïfung zum Abfallmanagement und zum Naturschutz sind hingegen Übergangsfristen vorgesehen.

Den zweiten Kernpunkt der Annäherungsstrategie der EU neben der Umsetzung der EU-Direktiven in nationales Recht bildet der Implementationsprozeß der Vorschriften. Damit sind zugleich die wesentlichen Problembereiche der Anpassung identifiziert (2).
Die Schätzungen der Anpassungskosten für die Übernahme des AC im Umweltbereich variieren erheblich. Eine Synthesestudie im Auftrag der EU kommt anhand der vorliegenden Studien zu dem Ergebnis, daß sich gemäß den vertrauenswürdigsten Daten die Investitionskosten auf ca. 120 Mrd. Euro belaufen werden, wobei insbesondere Investitionen in die Luftreinhaltung und Abwasserreinigung zu Buche schlagen (3). Allerdings ist bei diesen Zahlen zu berücksichtigen, daß für die zugrundeliegenden Studien unterschiedliche Methoden genutzt wurden, aufgrund fehlender Daten z.T. auf grobe Schätzungen zurückgegriffen werden mußte und zudem vielfach nicht die Differenz zu den EU-Standards, sondern die totalen Investitionskosten als Ausgangspunkt diente. Auch werden die Nutzen, die sich aus der Verbesserung der Umweltsituation ergeben, bei diesen Kostenschätzungen nicht erfaßt. Die Schätzungen entsprechen einer jährlichen Summe von 150 bis 200 Mark pro Kopf der Bevölkerung. Setzt man die Kosten in Relation zum Bruttoinlandsprodukt so wird deutlich, daß diese in den MOEL deutlich höher als in der EU-15 sind. Sie liegen sowohl im Hinblick auf Investitionen je Kopf als auch auf deren Anteil am BIP deutlich höher als bei der EU-Süderweiterung.

\section{Anpassungsprobleme}

Nach dem Subsidiaritätsprinzip obliegt die Gestaltung der politisch-administrativen Strukturen der mitgliedsstaatlichen Kompetenz. Zwar ist aufgrund des starken Implementationsdrucks eine Angleichung an den EU-Standard in den kommenden Jahren zu erwarten, allerdings müssen die administrativen Kapazitäten zur Durchsetzung des AC in den Schlüsselbereichen teilweise erst geschaffen werden. Damit wird auf wesentliche Schwachpunkte der Verwaltungen der Beitrittsstaaten verwiesen, wie die Fragmentierung des politisch-administrativen Systems, die mangelnde Politikkoordinierung, die
Abwanderung der qualifizierten Kräfte und fehlende Ressourcen (z.B. fehlende Mittelbereitstellung für Umweltagenturen).

Dennoch hat die Angleichung der nationalen Rechtsvorschriften im Umweltbereich an den Besitzstand der EU in allen fünf Ländern eingesetzt und zu erkennbaren Fortschritten gefïhrt. Probleme gibt es insbesondere bei Vorschriften in Bereichen, die aufgrund ihres infrastrukturellen Charakters hohe Investitionen erfordern, wie z.B. Trinkwasserversorgung und Abwasserbehandlung, Großfeuerungsanlagen und die Abfallbehandlung. Differenziert nach Bereichen ist die Übereinstimmung mit dem EU-Besitzstand bei der Verbesserung der Wasserqualität eindeutig am höchsten, im Durchschnitt für die fünf Länder lag sie 1995 bereits bei 70 Prozent der Regelungen. Bei der Bekämpfung der Luftverschmutzung und besonders im Abfallbereich liegt sie hingegen deutlich niedriger (4).

Die Europäische Union versucht, diese Entwicklungen zum einen mit know-how und zum anderen mit finanziellen Hilfen zu unterstïtzen. Den (begrenzten) finanziellen Rahmen hierfür stellt bislang noch immer vorrangig das PHARE-Programm dar, dessen Umsetzung allerdings aufgrund fragwürdiger Projekte starke öffentliche Kritik hervorgerufen hat (5). Technische Hilfe wird z.B. über das Technical Assistance Information Exchange Office (TAIEX) bereitgestellt. Auch bilaterale Beitrittspartnerschaften sollen zur Bewältigung der Probleme beitragen.

\section{Bewertung}

Für die MOEL ist die Übernahme des AC mit erheblichen, vor allem auch finanziellen Aufwendungen verbunden, ohne daß die Folgen durchgängig als positiv bezeichnet werden könnten (6). So ignoriert das Ziel der strikten Harmonisierung gerade die neuen Entwicklungen auf der EU-Ebene selbst, die das Ausmaß der Harmonisierung eher abschwächen (z.B. Auto-Öl- und IPPC-Richtlinie). Zudem wird damt die ordnungsrechtliche Prägung der Umweltpolitik übertragen und somit auf den Einsatz effizienterer ökonomischer Instrumente weitgehend verzichtet. Schließlich werden die durchaus auch vorhandenen ökologischen Pluspunkte der MOEL z.B. im Bereich ökologisch wertvoller Landschaften durch andere EU-Politikbereiche wie die Agrar- und Verkehrspolitik zumindest in Frage gestellt. 
Die EU-Osterweiterung stellt zugleich eine wesentliche Herausforderung für die Reformfähigkeit der EU dar. Dabei dürfte die Schönwetterperiode für die (erweiterte) EU vorüber sein. Dies gilt möglicherweise auch für die Umweltpolitik, die bei der Süderweiterung durch unterstützende Strukturfonds abgesichert werden konnte und bei der Norderweiterung durch die umweltpolitisch fortgeschrittenen Staaten eher gestïtzt wurde. Aus den neuen Beitrittstaaten erscheinen aktuell weder umweltpolitische Initiativen erwartbar, noch wird ihnen umfassende finanzielle Unterstützung gewährt werden. Eine solche wäre jedoch angesichts der gesamteuropäischen ökologischen Vorteile durch die Harmonisierung, der induzierten ökonomischen Belastungen der MOEL sowie der ökonomischen Vorteile insbesondere einiger Staaten der EU, für die der Beitritt einem Förderprogramm der Umwelttechnikindustrie gleichkommt, zumindest teilweise angemessen.

Ungeachtet dieser Einschränkungen stellen die Beitrittsverhandlungen für die MOEL einen wesentlichen Anreiz dar, trotz der vielfältigen anderen Transformationsprobleme Umweltaspekte nicht zu vernachlässigen.

\section{Anmerkungen}

(1) Vgl. Europäische Kommission: Agenda 2000, 1997.

(2) Vgl. für eine ausführliche Erörterung Ulrich Petschow/ Ralf Nordbeck/ Jan Nill: Der ProzeßB der EU-Osterweiterung aus umweltpolitischer Perspektive. IÖW-Schriftenreihe 137/98 (im Erscheinen).

(3) Vgl. De Nocker, Leo et al.: Compliance Costing for Approximation of EU Environmental Legislation in the CEEC. Report to DG XI, Brüssel, European Commission, 1997.

(4) Vgl. Regional Environmental Center: Approximation of European Union Environmental Legislation. Budapest 1996.

(5) Vgl. hierzu bereits Stein, Regine: Westliche Hilfsprogramme für die Länder Mittel- und Osteuropas im Umweltsektor - Note: ungenügend. In: IÖW/VÖW-Informationsdienst, Nr. 2/1994, S.16-17.

(6) $\mathrm{Vgl}$ z.B. Caddy, J.: Harmonisation and asymmetry: environmental policy co-ordination between the European Union and Central Europe. In: Journal of European Public Policy. Vol. 4 (1997), Nr. 3.

\section{Die Autoren}

Jan Nill und Ulrich Petschow sind Mitarbeiter des For schungsfelds Umweliökonomie und -politik des IÖW. Kontakt: IÖW, Giesebrechistr. 13, 10629 Berlin, Tel. 030/884594-0, Fax 030/ 8825439, E-mail: Jan.Nill@ioew.de

Verbändevereinbarung: Instrument zur Behinderung des Wettbewerbs mit sauberem Strom

\title{
Diskriminierende Praxis
}

\author{
Die deutsche Energiewirtschaft steht vor einem Umbruch: Dort wo jahrzehnte- \\ lang Monopole das Sagen hatten, soll umfassender Wettbewerb einkehren. Das \\ neve Energierecht (1) ermöglicht den Verbrauchern im Prinzip zu wählen, wel- \\ chen Strom sie in Zukunft einkaufen wollen. Abgesehen von seinen umwelt- \\ und klimapolitischen Defiziten wird das neue Recht vor allem wegen seiner \\ wettbewerbsverzerrenden Elemente kritisiert.
}

$\mathrm{K}$ ernpunkt des angestrebten europäischen Stromhandels ist der Netzzugang aller Anbieter und Abnehmer (Verbraucher). Sowohl in der EU-Stromrichtlinie von 1997 als auch im neuen deutschen Energierecht ist der diskriminierungsfreie Netzzugang gesetzlich festgeschrieben. Dennoch ist absehbar, daß es zukünftig Probleme geben wird, wenn z.B. ein kleinerer Newcomer Strom durch das Netz eines oder gar mehrerer Netzbetreiber-Elektrizitätsversorgungsunternehmen (EVU) leiten will. Im Rahmen einer freiwilligen Vereinbarung zwischen dem Bundesverband der Deutschen Industrie e.V., dem Verband der Industriellen Energieund Kraftwirtschaft und der Vereinigung Deutscher Elektrizitätswerke (VDEW), der sogenannten Verbändevereinbarung, werden Kriterien und Preise für den Netzzugang und die Bestimmung der Durchleitungsentgelte vorgeschlagen. Diese Vereinbarung ist für die Energieversorgungsunternehmen zwar nicht verbindlich, wird jedoch in Zukunft in den meisten Fällen für die Bestimmung des Durchleitungsentgelts herangezogen werden. Problematisch hierbei ist, daß die vorgesehene Regelung der im Energiewirtschaftsgesetz vorgeschriebenen Kostenorientierung nicht gerecht wird. Vielmehr wirkt die ungerechtfertigte Kostenzuweisung für bestimmte Durchleiter diskriminierend und führt zu Gewinnmitnahmen bei den netzbesitzenden Energieversorgungsunternehmen. Es ist sogar strittig, ob die Vereinbarung wettbewerbsverzerrend, und deshalb kartellrechtlich zulässig ist.

Es besteht zwar die Möglichkeit zum Erlaß einer Rechtsverordnung, die die Bestimmung transparenter und nichtdiskriminierender Durchleitungsbedingungen und -entgelte regeln könnte, aber sowohl Bundesregierung als auch Bundesratsmehrheit bauen bisher auf den Erfolg der Verbändevereinbarung.
Problematisch sind vor allem Regelungen, die die Durchleitung von Strom teuer machen bzw. erschweren. Die Durchschnittsnetzkosten enthalten Investitionen bzw. Abschreibungen des Kapitals, Wartung, Instandhaltung sowie ein Gewinnaufschlag. Diese Kosten fallen allerdings real bei der Durchleitung nicht an. Besonders bei der Durchleitung von Leistungen mit geringen Jahresbenutzungsstunden übersteigen die Durchleitungsgebühren die Erzeugungskosten im Kraftwerk teilweise um ein Mehrfaches.

Durchleitungsgebühren sollten jedoch für alle Akteure gleich, transparent sowie einfach und schnell handhabbar sein, um möglichst viele Wettbewerber in den Wettbewerb einbeziehen zu können. Zusätzlich sollten sie für die Netzbetreiber ökonomische Anreize sichern, damit sie trotz des natürlichen Monopols und der praktizierten Durchschnittskosten-Preisbildung an einer Effizienzverbesserung interessiert sind. In Großbritannien hat sich das sogenannte ,pricecap"-Prinzip, eine (obere) Begrenzung der Preisentwicklung, bewährt (2).

\section{Regenerative diskriminiert}

Die Durchleiter müssen für die Netzbenutzung für jeden Netzbereich und jede Netzebene Leistungskosten bezahlen. Dabei richtet sich das Entgelt an der höchsten Leistung ( der höchsten Strommenge bezogen auf die Zeiteinheit) aus, die der Durchleiter im Laufe eines Jahres mit seinem Abnehmer vereinbart bzw. liefert. Diese Regelung ist insbesondere für regenerative Energiequellen ungünstig, weil diese nur eine beschränkte Ausnutzungsdauer ihrer installierten Leistung pro Jahr haben. Es kann gezeigt werden, daß aufgrund der leistungsbezogenen Ermittlung der Durchleitungsgebühr etwa der dreifache Wert der tatsächlich im Durchschnitt anfallenden Netzkosten berechnet wird. 
(c) 20I0 Authors; licensee IÖW and oekom verlag. This is an article distributed under the terms of the Creative Commons Attribution Non-Commercial No Derivates License (http://creativecommons.org/licenses/by-nc-nd/3.o/), which permits unrestricted use, distribution, and reproduction in any medium, provided the original work is properly cited. 